\title{
Risk Perceptions and Behaviour: Towards Pandemic Control of Emerging Infectious Diseases
}

\author{
International Research on Risk Perception in the Control of Emerging Infectious \\ Diseases
}

\author{
Johannes Brug • Arja R. Aro • Jan Hendrik Richardus
}

Published online: 6 January 2009

(C) International Society of Behavioral Medicine 2008

In the beginning of 2003, the world was alarmed by the emergence of a new and apparently fatal infectious disease. The disease was labelled SARS. Thanks to enormous efforts made by national and international organisations, the epidemic was brought under control by the summer of that year. In recent years, the world has also been confronted with outbreaks or threats of outbreaks of other emerging infectious diseases such as avian influenza.

To control new infectious diseases, the identification of the organisms, the infectivity, development of vaccines and therapies, contact tracing, isolation and screening may all be important. Many of these issues are partly dependent on human behaviours. For example, the success of prevention of infectivity (e.g. engaging in precautionary behaviours such as wearing masks, hand hygiene, isolation etc.), vaccination, contact tracing and population screening are all more or less dependent on whether people at risk comply with

\footnotetext{
J. Brug $(\triangle)$

EMGO Institute, VU University Medical Centre,

Van der Boechorststraat 7 ,

1081 BT Amsterdam, The Netherlands

e-mail: j.brug@vumc.nl

J. Brug $\cdot$ J. H. Richardus

Department of Public Health, Erasmus MC,

University Medical Centre Rotterdam,

Rotterdam, The Netherlands

A. R. Aro

Unit for Health Promotion Research,

University of Southern Denmark,

Esbjerg, Denmark

J. H. Richardus

Municipal Health Service Rotterdam Rijnmond,

Rotterdam, The Netherlands
}

behavioural recommendations. Especially in the early phases of a possible epidemic, compliance to precautionary behaviours among the populations at risk is often the only means of prevention of a further spread of the disease. However, very little research has been conducted to explore the determinants of behavioural responses to infectious disease outbreaks $[1,2]$.

The present special series of the International Journal of Behavioral Medicine is dedicated to such research. One of the six papers, i.e. by Vartti et al. [3], in this special series originated from international collaboration of behavioural scientists to study risk perceptions around SARS during the SARS outbreak. The Aro et al. [4] paper represents early work related to risk perceptions among travellers during the avian influenza outbreak. Three papers [5-7] were the result of a European Commission funded project, called SARS-Control that was partly dedicated to exploring risk perceptions and risk communications related to SARS and other emerging infectious diseases.

\section{The SARS Epidemic and other Emerging Infectious Diseases}

Severe acute respiratory syndrome (SARS) was a new infectious disease due to an infection with a novel coronavirus, which was provisionally termed SARS-associated coronavirus (SARS-CoV) [8-10]. The earliest cases of SARS are known to have occurred in mid-November 2002 in Guangdong Province, China. SARS was first recognised in late February 2003, when cases of an atypical pneumonia of unknown cause began appearing among staff at hospitals in Guangdong, China and Hanoi, Vietnam [11]. Within 2 weeks, similar outbreaks occurred in various hospitals in Hong Kong, Singapore and 
Toronto, and the number of worldwide cases exceeded 4,000 within 2 months, and 7,000 a few weeks later, with cases being reported from 30 countries. During the peak of the global outbreak, near the start of May 2003, more than 200 new cases were being reported each day. More than 900 people died from SARS [12]. China was hit hardest, with over 5,000 patients and approximately 350 deaths. After July, SARS appeared to be under control.

Although SARS did not have the disastrous health impact that many at first feared, the panic caused by SARS had an enormous economic impact in many countries because of the health fears and related control measures. The global travel, tourism and related industries in particular faced a significant downturn in income, although mostly temporary. The global macroeconomic impact has been estimated at 30 to 100 billion US dollars. Although the European Union was not afflicted heavily by the SARS epidemic in terms of patient numbers, there was a large public concern related to the disease.

While the dissemination of SARS has been prevented in Europe and controlled in all affected areas within a few months, this may not be the case for other emerging infectious diseases. For instance, the West Nile virus was introduced in North America in 1999 and has been widely diffused since then despite very aggressive control efforts. In the USA in 2002, 4,156 cases were notified among whom 284 died [13]. Severe infection can emerge in Europe too. The H7N7 influenza episode among workers of the Dutch poultry industry in 2003 [14] has shown that the potential for pandemic influenza to start within Europe is there. Circulation of a human strain at the same time as the zoonotic strains (H7N7) were circulating in the poultry worker population could have precipitated the emergence of a new strain adapted to humans with fast secondary diffusion.

\section{International Research Projects}

Already in April 2003, an international Psychosocial SARS Research Consortium was formed initiated by professor George Bishop at Singapore University, which developed a survey instrument in several languages to probe awareness, knowledge, risk perceptions and precautionary behaviours related to SARS. In 2004, a European Union sponsored 3year research programme SARSControl was started with collaborators from Europe and China (partly building on the methods of the Psychosocial SARS Research Consortium) with the title "Effective and Acceptable Strategies for the Control of SARS and New Emerging Infections in China and Europe"; SARSControl. Risk perception and risk communication were themes in two out of nine work packages of the SARSControl project.

\section{The Importance of Risk Perception}

Effective management of new epidemic infectious disease risks in the phase that no treatment or vaccination is yet possible is largely dependent on precautionary behaviour of the population. Implementation of precautionary behaviour is largely dependent on effective risk communication, i.e. communication that induces realistic risk perceptions, correct knowledge and skills to promote and enable precautionary practices. Scientific knowledge about these topics in the area of infectious disease control is scarce. Neither is there knowledge if the theories and measures developed for risk perception research on, for example chronic diseases, can be applied in the area of infectious diseases. However, such knowledge is vital for effective control of newly emerging infectious diseases, because our ability to promote health protective behavioural change depends on our knowledge of important determinants of such behaviour [15]. For people to voluntarily engage in precautionary actions, they first of all need to be aware of the risk. Risk perception is a central feature in many health behaviour theories. According to the Protection Motivation Theory, for example [16], protection motivation is the result of the threat appraisal and coping appraisal. Threat appraisal consists of estimates of the chance of contracting a disease (perceived vulnerability or susceptibility) and estimates of seriousness of a disease (perceived severity).

Risk perceptions thus are important for precautionary actions, but risk perceptions are often biased [17]. Unrealistic optimism about health risks is often observed related to familiar risks that are perceived to be largely under volitional control. Such optimism may result in lack of precautions and false feelings of security. A pessimistic bias is more likely for new, unfamiliar risks that are perceived as uncontrollable. Such unwarranted high-risk perceptions may lead to unnecessary mass scares, and are often combined with stigmatisation of specific risk groups. Perceptions of risk are a necessary but often not sufficient condition for engagement is such behaviours. Therefore, higher risk perceptions may only predict protective behaviour when people believe that effective protective actions are available (response efficacy) and when they are confident that they have the abilities to engage in such protective actions (self-efficacy). Preliminary research on SARS as well as avian influenza risk perceptions support these theorised associations and show inverse associations between risk perceptions and efficacy beliefs $[2,18]$.

Furthermore, risk perceptions as well as efficacy beliefs in the early stages of a possible pandemic are dependent on communications with and between the members of the groups at risk. Risk communication messages that are not comprehended by the public at risk, or communication of conflicting risk messages will result in lack of precaution- 
ary actions. Communications that are perceived as coming from a non-trustworthy source may have the same results. However, risk communication messages are sometimes very quickly adopted by the media, possibly leading to an 'amplification' of risk information that may lead to unnecessary mass scares and unnecessary or ineffective precautionary actions.

\section{This Special Series}

In the first paper of this special series, Leppin and Aro [19] provide an overview of the theoretical frameworks on which risk perception and infectious disease research is founded. Leppin and Aro first of all make a distinction between a more sociological and a primarily psychological approach to risk perception research. They conclude that the current risk perception research in infectious disease epidemics is seldom theory based or conceptually clear. This is understandable when doing first surveys in the early phases of new emerging epidemics, but there certainly is a need to do consolidate the theoretical and methodological research base. We also need to find out empirically if the theories and methods developed mostly for chronic diseases under volitional control of individuals can be directly applied in emerging epidemics.

Four of the papers present empirical mostly explorative original research on risk perceptions, knowledge, beliefs and other issues related to SARS during or after the SARS outbreak in 2003. De Zwart and colleagues, in their eightcountry survey in 2005, almost 2 years after the SARS outbreak, found out that perceived threat of SARS in case of an outbreak in the country was higher than that of other diseases [7]. Perceived vulnerability of SARS was at an intermediate level compared to other diseases while perceived severity was high. Perceived threat for SARS varied between countries in Europe and Asia, but these differences did not appear to be associated with the proximity of the SARS 2003 outbreak.

Vartti et al. [3] in their study during the SARS outbreak found that despite the fact that both Finland and the Netherlands were unaffected by the outbreak the Finns were more likely to be knowledgeable and worried about SARS as well as to have low perceived comparative SARS risk and poor personal efficacy beliefs to prevent SARS. The Finns were also more likely to have high confidence in physicians in the SARS issues and less likely to have received information from the internet and have confidence in the internet information than the Dutch.

Voeten et al. [5] and Jiang et al. [6] studied the Chinese communities in the Netherlands and the UK because of their close communication and travel contacts with China, where the outbreak was the most severe. Jiang and colleagues, in their qualitative study, revealed that information from affected Asia influenced the perceived threat form SARS and protective behaviour among the Chinese in Europe when more relevant local information was absent. When a high perceived threat was combined with low efficacy regarding precautionary measures, avoidance-based precautionary action appeared to dominate responses to SARS. These actions may have contributed to the adverse impact of SARS on the community. The Voeten et al. [5] study results indicate that the Chinese community members relied more on information from friends and Chinese media and had less confidence in their doctor, government agencies and consumer interest groups. While their knowledge of SARS was high, they reported a lower perceived threat and higher self-efficacy than general populations with regard to SARS and avian flu, due to a lower perceived severity.

The Aro et al. [4] study, from the early phase of the avian influenza outbreak, found out that younger travellers and those on holidays are willing to take more health risks than those older or on business trips.

The overall results indicate that people across Europe and East Asia do regard recently emerging infectious diseases as serious potential health threats, based on information they receive from a range of different sources, with clear differences between countries and regions. These differences appear not to be necessarily associated with proximity of an outbreak. It remains unclear if cultural differences or experience with an outbreak may explain these differences in risk perceptions and beliefs.

Given the clear and present danger of newly emerging infectious disease outbreaks in the near future and the importance of the public response and precautionary actions to control the spread, additional research on risk perceptions and other behavioural determinants is warranted. The present series of papers present a first qualitative and social-epidemiological exploration. More theory-driven and stronger designed longitudinal and experimental studies are needed to test some of the hypotheses touched upon in this issue.

\section{References}

1. Smith RD. Responding to global infectious disease outbreaks: lessons from SARS on the role of risk perception, communication and management. Soc Sci Med. 2006;63:3113-23.

2. Brug J, Aro AR, Oenema A, de Zwart O, Richardus JH, Bishop GD. SARS risk perception, knowledge, precautions, and information sources, the Netherlands. Emerg Infect Dis. 2004;10(8):1486-9.

3. Vartti AM, Oenema A, Schreck M, Uutela A, de Zwart O, Brug J, et al. SARS knowledge, perceptions and behaviors: a comparison between Finns and the Dutch during the SARS outbreak in 2003. Int J Behav Med 2009;16. doi:10.1007/s12529-008-9004-6.

4. Aro AR, Vartti AM, Schreck M, Turtianen P, Uutela A. Willingness to take travel-related health risks: a study among 
Finnish tourists in Asia during the avian influenza outbreak. Int J Behav Med 2009;16. doi:10.1007/s12529-008-9003-7.

5. Voeten HACM, de Zwart O, Veldhuijzen IK, Yuen C, Jiang X, Elam G, et al. Sources of information and health beliefs related to SARS and avian influenza among Chinese communities in the United Kingdom and the Netherlands, as compared to the general population in these countries. Int J Behav Med 2009;16. doi:10.1007/s12529-008-9006-4.

6. Jiang X, Elam G, Yuen C, Voeten H, de Zwart O, Veldhuijzen I, et al. The perceived threat of SARS and its impact on precautionary actions and adverse consequences: a qualitative study among Chinese communities in the United Kingdom and the Netherlands. Int J Behav Med 2009;16. doi:10.1007/s12529008-9005-5.

7. de Zwart O, Veldhuijzen I, Elam G, Aro AR, Abraham T, Bishop $\mathrm{GD}$, et al. Perceived threat, risk perception and efficacy beliefs related to SARS and other (emerging) infectious diseases: results of an international survey. Int J Behav Med 2009;16. doi:10.1007/ s12529-008-9008-2.

8. Peiris JS, Lai ST, Poon LL, Guan Y, Yam LY, Lim W, et al. Coronavirus as a possible cause of severe acute respiratory syndrome. Lancet. 2003;361(9366):1319-25.

9. Ksiazek TG, ErdmanD, Goldsmith CS, Zaki SR, Peret T, Emery $\mathrm{S}$, et al. A novel coronavirus associated with severe acute respiratory syndrome. N Engl J Med. 2003;348(20):1953-66.

10. Drosten C, Günther S, PreisterW, van der Werf S, Brodt HR, Becker $\mathrm{S}$, et al. Identification of a novel coronavirus in patients with severe acute respiratory syndrome. N Engl J Med. 2003;348 (20):1967-76.

11. Kamps BS, Hoffman C (2003) SARSReference.com 10/2003, Flying Publisher [On-line]. Available: www.sarsreference.com.

12. World Health Organization (2003). Cumulative Number of Reported Cases of Severe Acute Respiratory Syndrome (SARS) [On-line]. Available: http://www.who.int/csr/sars/country.

13. Perkins LE, Swayne DE. Comparative susceptibility of selected avian and mammalian species to a Hong Kong-origin H5N1 highpathogenicity avian influenza virus. Avian Dis. 2003;47(3 Suppl):956-67.

14. Hayden F, Croisier A. Transmission of avian influenza viruses to and between humans. J Infect Dis. 2005;192(8):1311-4.

15. Brug J, Oenema A, Ferreira I. Theory, evidence and Intervention Mapping to improve behavior nutrition and physical activity interventions. Int J Behav Nutr Phys Activ. 2005;2:2.

16. Rogers RW. Cognitive and physiological processes in fear appeals and attitude change: a revised theory of protection motivation. New York: Guilford; 1983.

17. Weinstein ND. The precaution adoption process. Health Psychol. 1988;7(4):355-86.

18. de Zwart O, Veldhuijzen IK, Elam G, Aro AR, Abraham T, Bishop GD, et al. Avian flu risk perception: Europe and Asia. Emerg Infect Dis. 2007;13:290-3.

19. Leppin A, Aro AR. Risk perception related to SARS and avian influenza: theoretical foundations of current behavioral research. Int J Behav Med 2009;16. doi:10.1007/s12529-008-9002-8. 